\title{
Correlation between increased serum malondialdehyde and spectrum of cranial ultrasonography findings in hypoxic ischemic encephalopathy: could it be used as a predictor of disease severity?
}

Hadeel M. Seif El Dein ${ }^{1 *}$, Nouran Fahmy ${ }^{2}$, Zahraa Ezz El Din², Marianne Morgan ${ }^{3}$, Marwa Abdel Fattah² and Sara S. Eltatawy ${ }^{2}$

\begin{abstract}
Background: Hypoxic ischemic encephalopathy (HIE) is a major cause of mortality and morbidity in neonates. Malondialdehyde $(\mathrm{MDH})$ is a colorless lipid that can be used as a marker for oxidative stress. Cranial ultrasound sensitivity and specificity in detection of neonatal HIE ought to be further investigated. This study aims to detect whether serum (MDH) can be used as an indicator for HIE severity and to assess the role of cranial ultrasound in diagnosis of HIE neurological disorders, correlating ultrasound findings to MDA levels.

Results: Statistically significant differences were found between the serum MDA levels in patients compared to controls as well as among serum MDA in patients with advancing Sarnat stages (I, II, III) $P$ value $<0.001$. Statistically significant levels of serum MDA were found in patients with ischemic US findings compared to those with normal scan; $36.4 \%$ of cases with ischemic US findings were diagnosed as Sarnat stage II while 63.6\% were diagnosed with stage III with a statistically significant difference $(P=0.016)$.

Conclusion: Cranial ultrasound can be used for diagnosis of neonatal hypoxic ischemic insults, with lower sensitivity in mild cases and increased sensitivity in severe cases; and when combined with measuring serum MDA levels, it can be used as a diagnostic marker and as a predictor for severity of HIE.
\end{abstract}

Keywords: Hypoxic ischemic encephalopathy (HIE), Serum malondialdehyde (MDH), Cranial ultrasound

\section{Background}

Hypoxic ischemic encephalopathy (HIE) remains one of the major causes of mortality and morbidity in neonates [1]. Having a reliable biomarker to diagnose and predict the severity of this condition is a valuable tool in the clinical setting. Malondialdehyde (MDA) is a colorless lipid resulting from the effect of free oxygen radicals on tissues and from a series of reactions during lipid

\footnotetext{
*Correspondence: Hadeel_mseif1@hotmail.com

${ }^{1}$ Department of Radiology, Cairo University, Cairo, Egypt

Full list of author information is available at the end of the article
}

peroxidation [2]. Serum MDA concentrations vary with varying severity of HIE and could therefore be used as a predictor for determining HIE staging, treatment and prognosis [3]. Cranial ultrasound is an easily acquired bed-side, radiation-free examination; its sensitivity and specificity in detection of neonatal HIE needs to be further investigated [4].

The aim of our study was to detect the role of serum malondialdehyde (MDA) levels in HIE and to determine whether MDA can be used as an indicator of severity in

\section{Springer Open}

(๑) The Author(s). 2020 Open Access This article is licensed under a Creative Commons Attribution 4.0 International License, which permits use, sharing, adaptation, distribution and reproduction in any medium or format, as long as you give appropriate credit to the original author(s) and the source, provide a link to the Creative Commons licence, and indicate if changes were made. The images or other third party material in this article are included in the article's Creative Commons licence, unless indicated otherwise in a credit line to the material. If material is not included in the article's Creative Commons licence and your intended use is not permitted by statutory regulation or exceeds the permitted use, you will need to obtain permission directly from the copyright holder. To view a copy of this licence, visit http://creativecommons.org/licenses/by/4.0/. 
HIE. We also aimed to detect MDA levels in correlation to cranial ultrasound findings in HIE patients.

\section{Methods}

This prospective study was carried out in the Neonatal Intensive Care Unit (NICU) Maternal Hospital. Patient collection spanned over a period of 2 years starting September 2014. It included 2 groups; a study group $(n=$ $42)$ and a control group $(n=42)$ adding up to a total of 84 subjects. The study group included 42 full-term and near-term (35-37 weeks) neonates who fulfilled the eligibility criteria for hypoxic ischemic encephalopathy (HIE). The control group, collected from the delivery room included 42 healthy neonates, matched for gestational age, gender, and birth weight.

Eligibility criteria for the stuFdy (hypoxic) group included near or full-term newborns delivered with indicators of peripartum hypoxia-ischemia and moderate to severe encephalopathy. Hypoxia-ischemia was defined as patients with Apgar scores less than 3 at $1 \mathrm{~min}$ and 5 at 5 min of life, arterial $\mathrm{pH}$ of $\leq 7.2$, base deficit $>-10$ mmol within $60 \mathrm{~min}$ of birth, and delayed first cry beyond $1 \mathrm{~min}$ of birth [5]. Encephalopathy was defined according to Sarnat criteria (lethargy, stupor, coma, abnormal tone, and/or seizures) [6]. The diagnosis of hypoxic ischemic encephalopathy was made by Neonatal ICU staff and attending physicians.

Infants were excluded from the study group if they were less than 35 weeks gestational age, had major congenital malformations, did not show the required evidence of hypoxia-ischemia at birth, or presented with hyperbilirubinemia.

The study was approved by local ethical committee and informed parental consent was given by all the patients in both groups. The following data were collected by NICU staff; perinatal history, gestational age using new Ballard score [7], birth weight, gender, mode of delivery, 1- and 5-min Apgar scores, and time of first cry. All patients were subjected to assessment of serum MDA within $24 \mathrm{~h}$ of life; the normal value of MDA using ELISA technique was defined as levels from 0.2 up to $1 \mathrm{nmol} / \mathrm{ml}[8]$.

Patients in the study hypoxic group were subjected to additional investigations including arterial blood for $\mathrm{pH}$, complete blood picture $(\mathrm{CBC})$, kidney function tests, liver function tests, coagulation profile, serum electrolytes, calcium levels, and random blood sugar. They underwent a clinical grading of neurological affection according to Sarnat and Sarnat Score after birth [6]. Other data such as presence of multisystem affection and need for inotropes was also recorded.

Due to early mortality, cranial ultrasound was only performed on 37/42 hypoxic group patients during their first week of life. This was done using the US machine
TOSHIBA with multifrequency probe (5-10 $\mathrm{MHz})$ through the anterior fontanel. Ischemic ultrasound findings were then correlated to MDA levels and clinical factors. Hypoxic ischemic affection was defined by ultrasound when the following findings were seen: brain edema with echogenic subcortical white matter, increased cerebral echogenicity with or without loss of gray-white matter differentiation, intraventricular bleeding, and/or thalamic or basal ganglia involvement [9]. Head sonography was also used to assess pulsed Doppler flow velocities and the resistive index of the cerebral arteries [10].

\section{Statistical methods}

Data were analyzed using IBM๑ SPSS $\odot$ Statistics version 23 (IBM@ Corp., Armonk, NY, USA), MedCalcœ version 15 (MedCalc@ Software bvba, Ostend, Belgium). Normality of numerical data distribution was examined using the D'Agostino-Pearson test.

Discrete numerical variables were presented as median and interquartile range and intergroup differences were compared using the Mann-Whitney test.

Continuous numerical variables were presented as mean $\pm \mathrm{SD}$ and intergroup differences were compared using the unpaired $t$ test (for two-group comparison) or one-way analysis of variance (ANOVA) (for multiplegroup comparison). The Tukey-Kramer post hoc test was used for pairwise comparison whenever the ANOVA test revealed a statistically significant difference among the groups.

Categorical variables were presented as number or proportion and percentage, and intergroup differences were compared using Fisher's exact test.

Receiver-operating characteristic (ROC) curve analysis was used to examine the predictive value of serum MDA. The area under the various ROC curves (AUC) was compared with that of random prediction using the DeLong method.

Correlations among numerical variables were tested using the Spearman rank correlation. Time to event analysis was done using the Kaplan-Meier method, and the log-rank test was used to compare individual KaplanMeier curves.

A two-sided $P$ value $<0.05$ was considered statistically significant.

Results

In our study of 84 neonates, female to male ratio of the hypoxic group were $36 \%$ and $64 \%$, respectively, while in the control group, gender was divided equally at $50 \%$. Mean gestational age of hypoxic and control groups was $38.2 \pm 1.4$, and $38.0 \pm 0.9$, respectively. Mean birth weight of hypoxic and control groups was $3.07 \pm 0.64$ and $3.09 \pm 0.4$, respectively. Mode of delivery in the hypoxic group was $57 \%$ CS and $43 \%$ vaginal delivery, while 
the control group was born $59.5 \%$ by CS and $40.5 \%$ by vaginal delivery.

Among maternal risk factors in our hypoxic group, hypertension proved to be the highest percentage at $33 \%$ followed by antepartum hemorrhage at $24 \%$, although neither showed a statistically significant difference.

In terms of hypoxic events surrounding the birth, perinatal hypoxia showed a highly significant difference of Apgar scores at 1 and 5 min between hypoxic group and control group $(P<0.001)$.

As for encephalopathy, the most common neurological manifestations in the hypoxic group were seizures (88\%) followed by hypotonia (74\%), hyporeflexia $(55 \%)$, disturbed conscious level (52\%) irritability (24\%), hyperreflexia (26\%) and hypertonia (2\%), which translated to $23 \%$ of hypoxic babies displaying Sarnat stages 1 and 2 and $52 \%$ displaying Sarnat stage 3

Multisystem affection is common in HIE babies; in our study, renal dysfunction was most frequent (99\%) followed by metabolic acidosis (91\%), pulmonary hypertension (60\%), hypotension (52\%), poor perfusion (48\%), MAS (41\%), bleeding tendency (38\%), and hypoglycemia (10\%). The most common complication in the hypoxic group was sepsis (43\%) followed by pneumonia (12\%). Outcome was unfavorable with a $43 \%$ mortality rate.

Our aim was to discover the role of MDA in HIE and correlate serum levels with severity of HIE. We found a highly significant difference between MDA levels in hypoxic neonates $(3.40 \pm 1.02) \mathrm{nmol} / \mathrm{ml}$ compared to the control group $(0.77 \pm 0.17) \mathrm{nmol} / \mathrm{ml}(P<0.001)$ (Fig. 1) with a ROC curve showing MDA level sensitivity and specificity of $100 \%$ in hypoxic neonates compared to control group. Area under curve was 1 with highly significant difference $(P<0.001)$ (Fig. 2$)$.

As for severity of HIE, higher MDA levels correlated to presence and earlier onset of seizures in the hypoxic group (Table 1). A cut-off level of $2.4 \mathrm{nmol} / \mathrm{ml}$ for MDA showed $84 \%$ sensitivity and $100 \%$ specificity for occurrence of seizures. Concerning timing of seizures in hypoxic babies, our data shows that at a level of $\leq 2.88$ $\mathrm{nmol} / \mathrm{ml}$, probability of occurrence of seizures before 12 $\mathrm{h}$ of life is extremely low (Fig. 3).

Mean MDA levels were $2.16 \pm 0.31 \mathrm{nmol} / \mathrm{ml}, 3.02 \pm$ $0.67 \dagger \mathrm{nmol} / \mathrm{ml}$, and $4.13 \pm 0.66+\ddagger \mathrm{nmol} / \mathrm{ml}$ correlating to Sarnat stages I, II, and III, respectively with a high statistically significant difference $(P<0.001)$ (Table 2$)$

ROC curves demonstrated a cut-off MDA level of $>$ $2.88 \mathrm{nmol} / \mathrm{ml}$ between Sarnat stage I and Sarnat stage II/III in hypoxic patients; area under the curve was 0.969 with a $90 \%$ sensitivity and $100 \%$ specificity (Fig. 4). As for discrimination between Sarnat stage III and Sarnat stage I/II, cut-off level for MDA was $>3.1 \mathrm{nmol} / \mathrm{ml}$; area under the curve was 0.942 with a $96 \%$ sensitivity and $85 \%$ specificity (Fig. 5). Finally, MDA levels correlated with outcome as well as severity; those with MDA levels $>2.88 \mathrm{nmol} / \mathrm{ml}$ had a longer hospital stay and were less likely to survive (Table 3 ).

In this study, cranial ultrasound was conducted for 37/ 42 hypoxic neonates and revealed 21/37 normal scans,

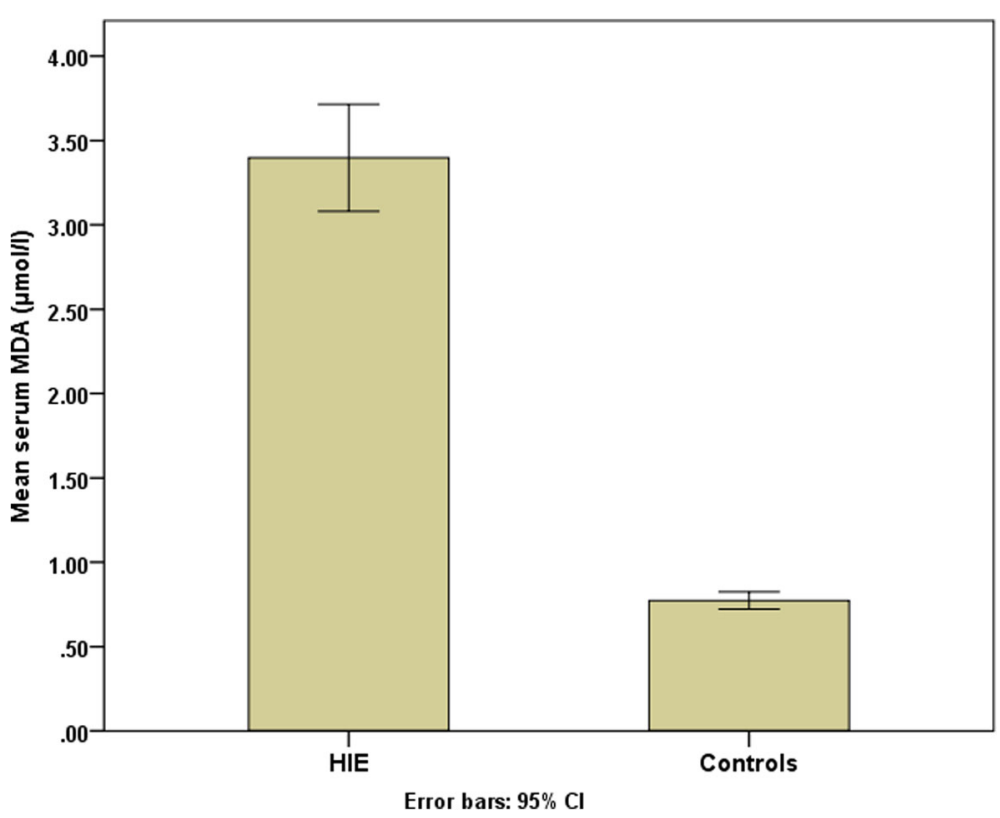

Fig. 1 Mean serum (MDA) levels in hypoxic neonates and control groups 


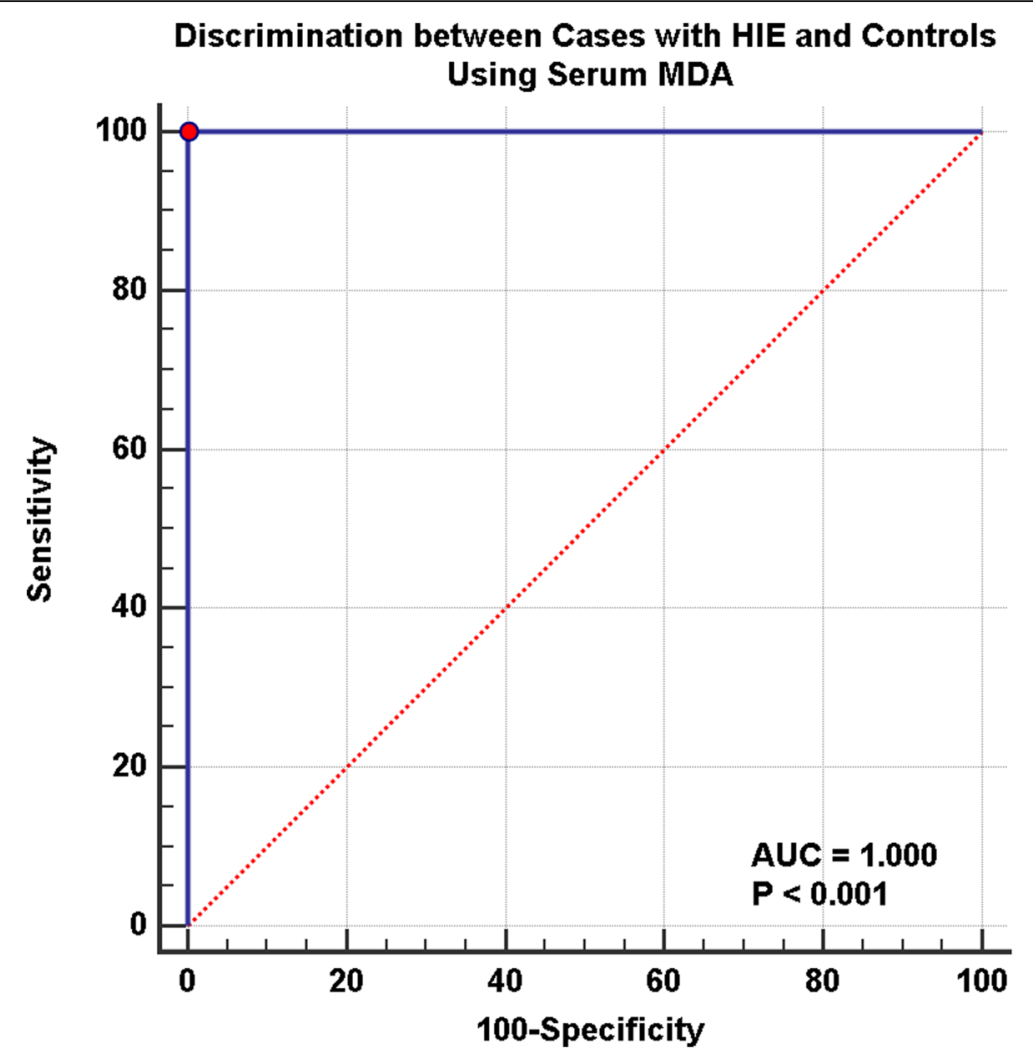

Fig. 2 ROC curve for discrimination between neonates with HIE and controls using serum (MDA) levels

while abnormal cranial US findings were found in 16 patients. We divided these 16 positive scans into ischemic pathology related to HIE (11/16) and non-ischemic (5/16). Ischemic cases included 4 with persistent diffuse subcortical hyperecchogenicities, 3 with unilateral thalamic echogenic foci, 2 with increased ecchogenicity and diffuse basal ganglia with brain edema, and 2 with focal subcortical white matter echogenic focus. Non-ischemic cranial ultrasound findings included 3 with obstructive hydrocephalus, and 2 with corpus callosum agenesis (Figs 6, 7, 8, and 9)

There was a significant difference between positive ischemic cranial ultrasound findings and Sarnat stages $(P$ $=0.016)$. The majority of positive ischemic US findings were present in Sarnat stage $3(63 \%)$ and Sarnat stage 2 (37\%) (Table 4).
As for correlation of HIE babies and cranial ultrasound findings, MDA levels were significantly higher in hypoxic neonates with positive ischemic US findings compared to those with negative US findings $(P$ value $=0.018$ ) (Table 5). Cut-off MDA level in hypoxic neonates with positive ischemic US findings was $>2.88 \mathrm{nmol} / \mathrm{ml}$. Sensitivity of MDA in positive ischemic US findings was $90.9 \%$ and specificity was $57.14 \%$ (Fig. 10).

\section{Discussion}

Hypoxic ischemic encephalopathy (HIE) remains a problem of great concern worldwide especially in developing countries. The occurrence of a neurological syndrome can be an indicator of an insult to the brain. HIE is an

Table 1 Relationship between MDA and occurrence of seizures

\begin{tabular}{|c|c|c|c|c|c|}
\hline Variable & Seizures $(n=37)$ & No seizures $(n=5)$ & $T$ & df & $P$ valuef \\
\hline Serum MDA(nmol/ml) & $3.57 \pm 0.96$ & $2.12 \pm 0.18$ & 8.166 & 35.662 & $<0.001$ \\
\hline
\end{tabular}

Data are mean \pm SD

qUnpaired $t$ Test

$t$ T statistic, $d f$ Degree of freedom 


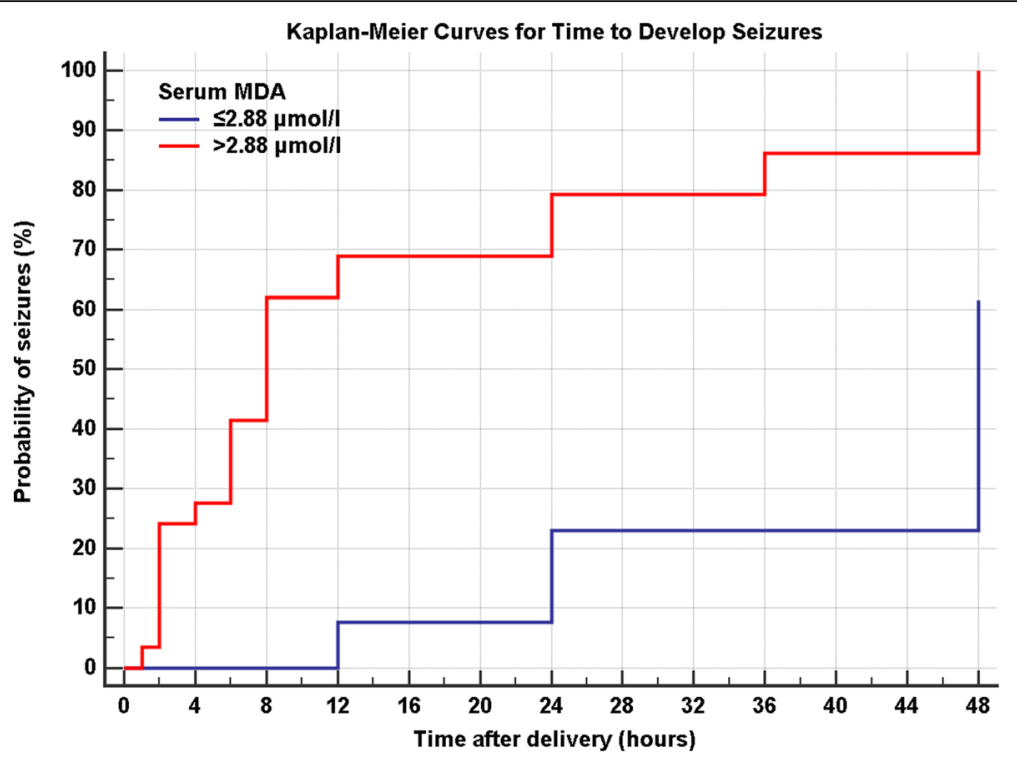

Fig. 3 Kaplan-Meier curves for time to develop seizures in patients with serum MDA $\leq 2.88 \mathrm{nmol} / \mathrm{ml}$ or $>2.88 \mathrm{nmol} / \mathrm{ml}$

important cause of perinatal mortality and lifelong neurodevelopmental morbidity [11].

This was a prospective study conducted on 84 neonates in the period between September 2014 and August 2016. The study included 42 newborns fulfilling the criteria of hypoxia ischemia as a patient group and 42 normal newborns as a control group.

In terms of mode of delivery, studies have shown disparity regarding its relation to HIE $[12,13]$. The timing of HIE may have a role in the variability of study results, whether the HIE was originally antepartum or whether it occurred as part of a difficult or complicated delivery; which may be a reason for varying conclusions on this point. Our study similar to another study by Alireza and Shirin [14] showed no statistical significance between the two groups in terms of mode of delivery.

Concerning hypoxic ischemic perinatal events, we found a highly significant difference regarding Apgar scores at 1 and 5 min compared to the control group $(P$
$<0.00)$. These results stand in accordance with studies done by Nicole et al. and Florio et al. [15, 16], who reported that Apgar scores at 1 and 5 min were significantly lower in hypoxic than control newborns. In another study, Dede [17] and his colleagues mentioned that there was a significant difference between hypoxic and control groups regarding Apgar score but only at 1 min. Apgar score still remains an important tool in newborn assessment and monitoring the progress of resuscitation in a resource-poor setting. Both the 1st and 5th min Apgar scores showed a good correlation with clinical features of severe forms of HIE [18].

Median PH for the hypoxic group in this study was 7.14 and median base excess was $-14 \mathrm{mmol} / \mathrm{L}$. Other studies have also found that profound metabolic alterations were present in hypoxic babies as compared to controls, whether in $\mathrm{pH}$ [3] and/or BE [19]. This was explained by Shah et al. [20] by demonstrating that there was a rise in organ injury rate as the arterial $\mathrm{pH}$ values

Table 2 Relation between MDA and Sarnat scores shows that the mean MDA levels were $2.16 \pm 0.31 \mu \mathrm{mol} / \mathrm{l}, 3.02 \pm 0.67+\mu \mathrm{mol} / \mathrm{l}$, and $4.13 \pm 0.66+\ddagger \mu \mathrm{mol} / \mathrm{I}$ regarding Sarnat stages I, II, and III, respectively, in hypoxic neonates with highly statistical significant difference $(P<0.001)$

\begin{tabular}{lllllll}
\hline Variable & Sarnat stage I & Sarnat stage II & Sarnat stage III & F & df & $P$ valuef \\
\hline $\mathrm{MDA}(\mathrm{nmol} / \mathrm{ml})$ & $2.16 \pm 0.31$ & $3.02 \pm 0.67 \dagger$ & $4.13 \pm 0.66+\ddagger$ & 39.491 & 2,39 & $<\mathbf{0 . 0 0 1}$ \\
\hline
\end{tabular}

Data are mean \pm SD

$F$ F statistic, $d f$ Degree of freedom

१One-way analysis of variance (ANOVA)

t $P$ value $<0.05$ vs. Sarnat stage I (Tukey-Kramer test)

$\ddagger P$ value $<0.05$ vs. Sarnat stage II (Tukey-Kramer test) 


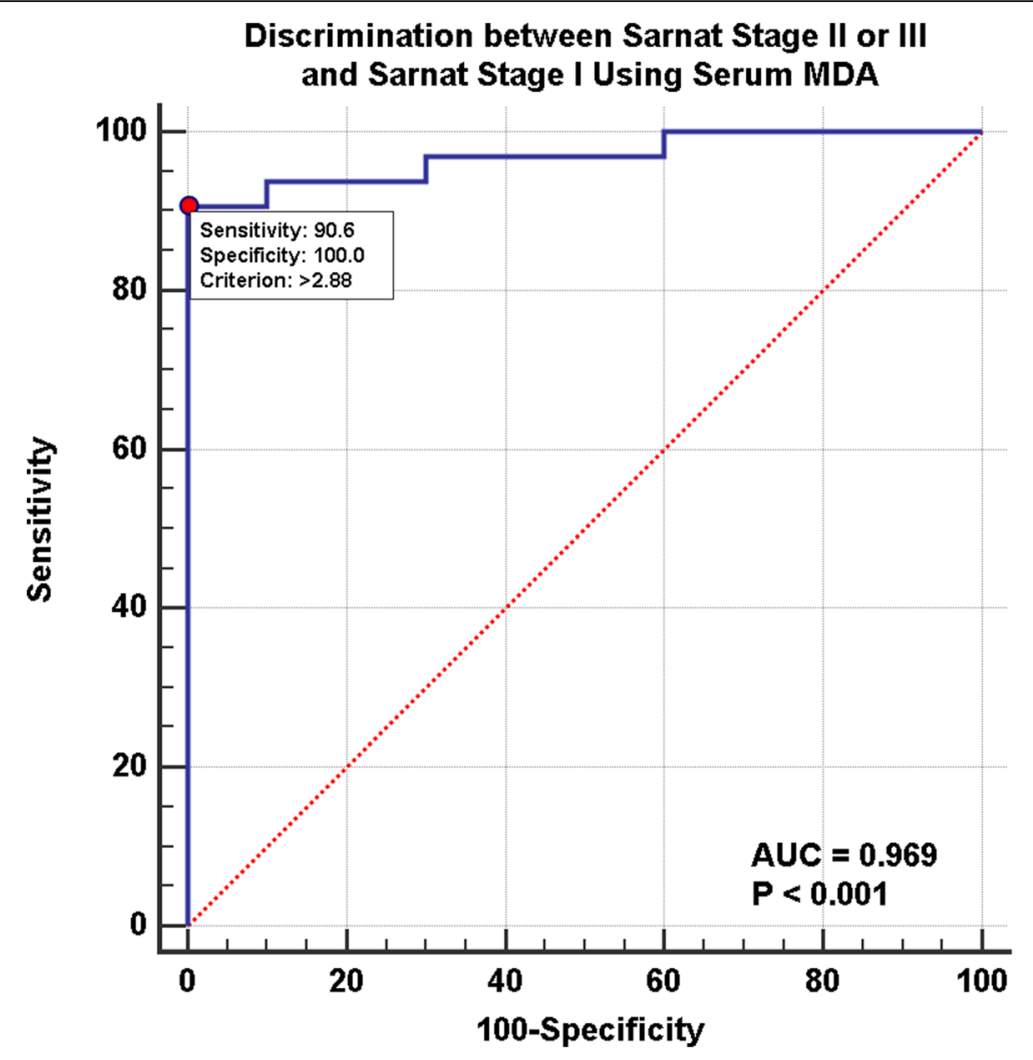

Fig. 4 ROC curve analysis for discrimination between Sarnat stage II or III and Sarnat stage I patients using serum MDA levels. Cut-off MDA level is $>2.88 \mathrm{nmol} / \mathrm{ml}$ and area under the curve is 0.969 . Sensitivity of MDA in Sarnat II or III compared to stage I was $90.62 \%$ and sensitivity was $100 \%$

decreased and base excess value dropped due to the fact that hypoxia causes anaerobic metabolism with high incidence of metabolic acidosis resulting in organ damage.

Hypoxia and ischemia can cause damage to almost every tissue and organ of the body and various target organs are involved. Gupta et al. [21] have reported injury to the kidneys in $50 \%$, followed by central nervous system, cardiovascular system, and lungs. The most common system affection in our study was renal dysfunction (99\%) followed by metabolic acidosis (91\%) then pulmonary hypertension (60\%). This is in agreement with another Egyptian study done by Mohammed et al. [22] who revealed that renal dysfunction was the most common followed by pulmonary dysfunction.

Since malondialdehyde (MDA) is one of the reactive metabolic products resulting from the effect of free oxygen radicals on tissues and from a series of reactions during lipid peroxidation [2], it can therefore, be used as a predictor for determining the presence and severity of HIE [3]. In our study, serum MDA levels were highly significant in hypoxic neonates $(3.40 \pm 1.02) \mathrm{nm} / \mathrm{ml}$ compared to the control group $(0.77 \pm 0.17) \mathrm{nm} / \mathrm{ml}[P<$ $0.001)$. This is in agreement with many other studies that showed MDA levels in hypoxic babies were highly significant compared to the control groups [23-25], indicating an association between perinatal asphyxia and oxidative stress.

In this study, there was a highly significant positive correlation between MDA and grading of encephalopathy; MDA levels were significantly higher in Sarnat stages II and III compared to Sarnat stage I $(P<0.001)$ as was mentioned by Kirimi et al. [24]. We also found a statistically significant positive correlation between rising MDA levels and progressive severity of encephalopathy as supported by Banupriya et al. [26]. Concerning neurological symptoms, as is mentioned in other studies [27], we similarly found serum MDA to be significantly higher in the patients who developed seizures compared to those who did not, highlighting the increasing severity of neurological symptoms with rising MDA levels.

Cranial US can be used to detect hemorrhage, periventricular leukomalacia (PVL), edema, and hydrocephalus [28]. The most common head sonography findings in neonates with hypoxic-ischemic injury are brain edema with echogenic subcortical white matter [29], which is consistent with finding by Van Wezel- 


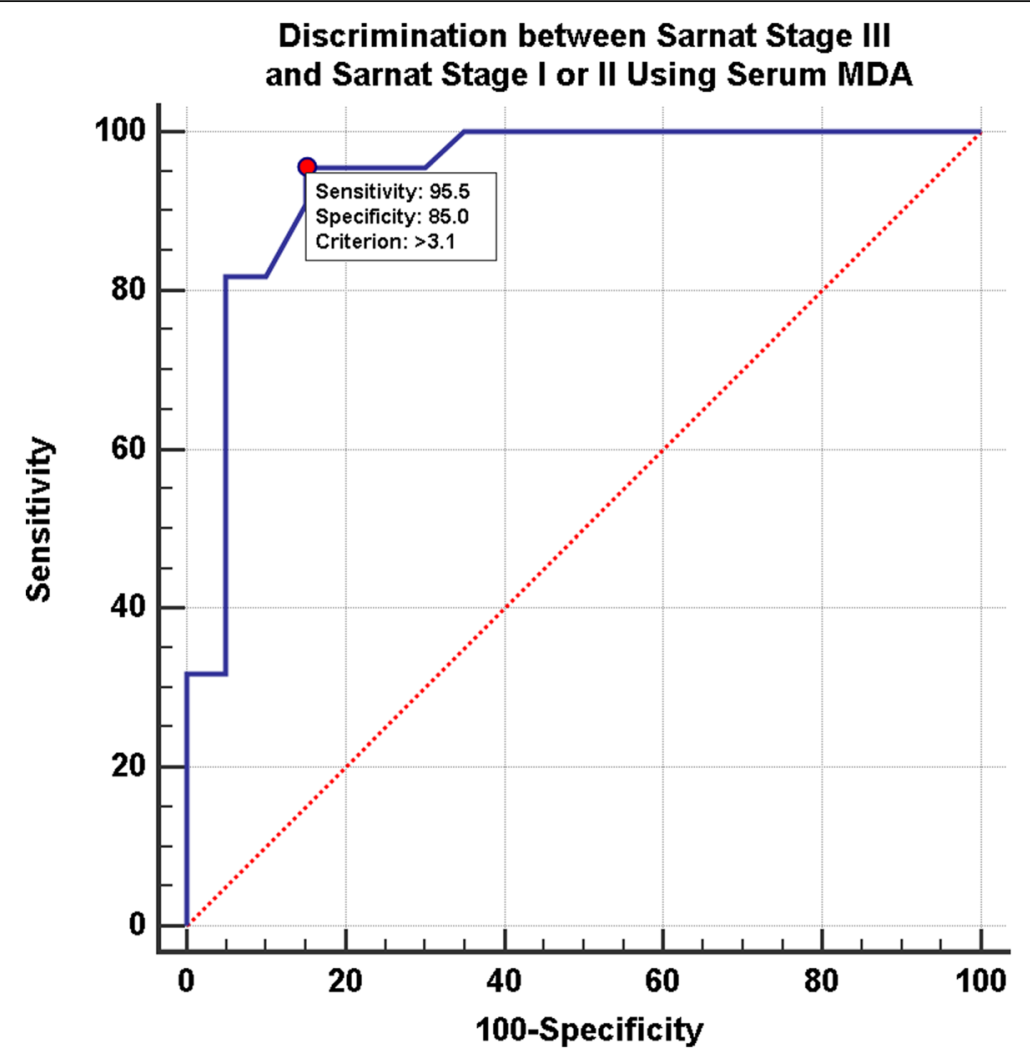

Fig. 5 ROC curve for discrimination between Sarnat stage III and Sarnat stage I or II patients using serum MDA levels. Area under the curve was 0.942. Cut-off MDA levels was > $3.1 \mathrm{nmol} / \mathrm{ml}$. Sensitivity of MDA in Sarnat stage III compared to stages II or I was $95.45 \%$ and specificity was $85 \%$

Meijler [30], as well as findings in our study. Another study found intracranial hemorrhage and diffuse cerebral edema to be the most common ultrasound finding in HIE [31].

We found that MDA levels were significantly higher in hypoxic neonates with positive ischemic US findings compared to those with negative US findings with a cutoff MDA value of $>2.88 \mathrm{nmol} / \mathrm{ml}$. We found a significant difference between positive ischemic cranial ultrasound finding and Sarnat stages; the majority of ischemic US findings were present in Sarnat stages II and III, which reveals a significant correlation between MDA, neurological deficits, and radiological findings of hypoxic neonates.
A cut-off level is essential to create a marker for clinicians to use for diagnosis and management of their patients. The significant MDA cut-off level that reappeared in our study was $2.88 \mathrm{nmol} / \mathrm{ml}$. This number was significant for discrimination of Sarnat stages higher than I, earlier onset of seizures, positive cranial ultrasound hypoxic ischemic findings, longer hospital stay, and higher chances of mortality.

One of the limitations of this study was the inability to perform brain MRIs for these patients since our facility does not have a portable machine, and transporting these critically ill babies was not feasible. A larger study with a bigger sample population would perhaps have given more weight to our work but

Table 3 Relation between MDA and survival

\begin{tabular}{llllll}
\hline Variable & Survivors $(\boldsymbol{n}=\mathbf{2 4})$ & Non-survivors $(\boldsymbol{n}=\mathbf{1 8})$ & $\mathbf{t}$ & df & $\boldsymbol{P}$ value \\
\hline Serum MDA $(\mathrm{nmol} / \mathrm{ml})$ & $2.94 \pm 0.96$ & $4.00 \pm 0.76$ & -3.864 & 40 & $<\mathbf{0 . 0 0 1}$
\end{tabular}

Data are mean \pm SD

$t$ T statistic, $d f$ Degree of freedom

Unpaired $t$ Test 

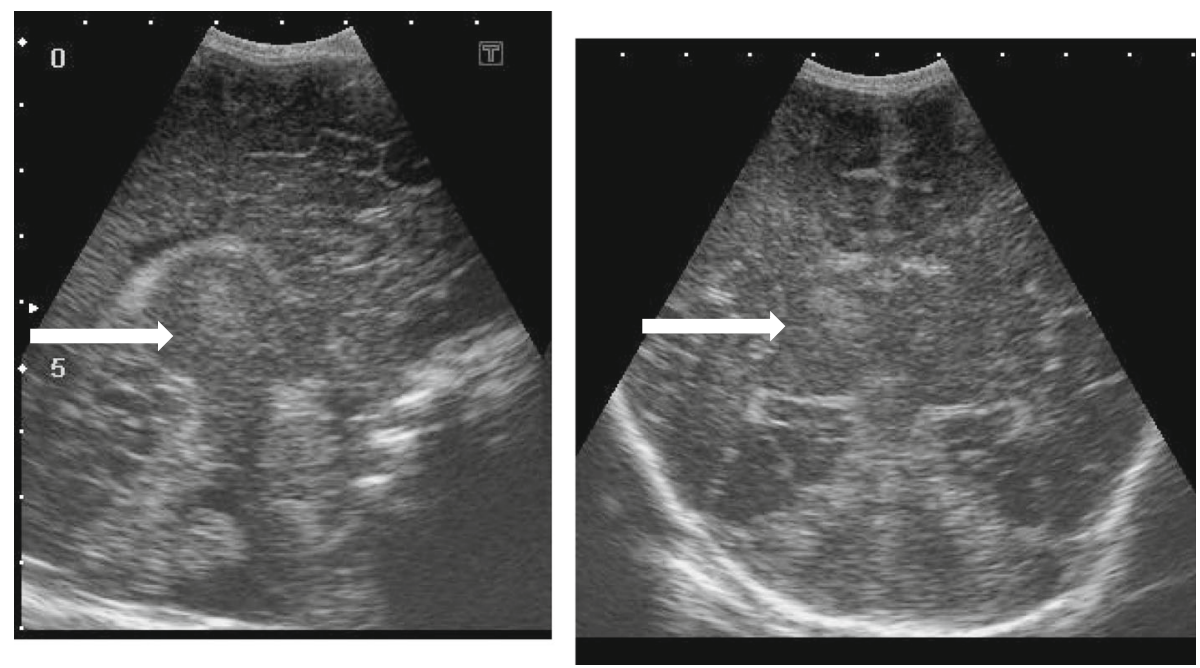

Fig. 6 Sagittal and coronal US image with Sarnat stage II showing right thalamic and smaller left thalamic focal echogenic areas (white arrow), representing thalamic hypoxic insult lesions

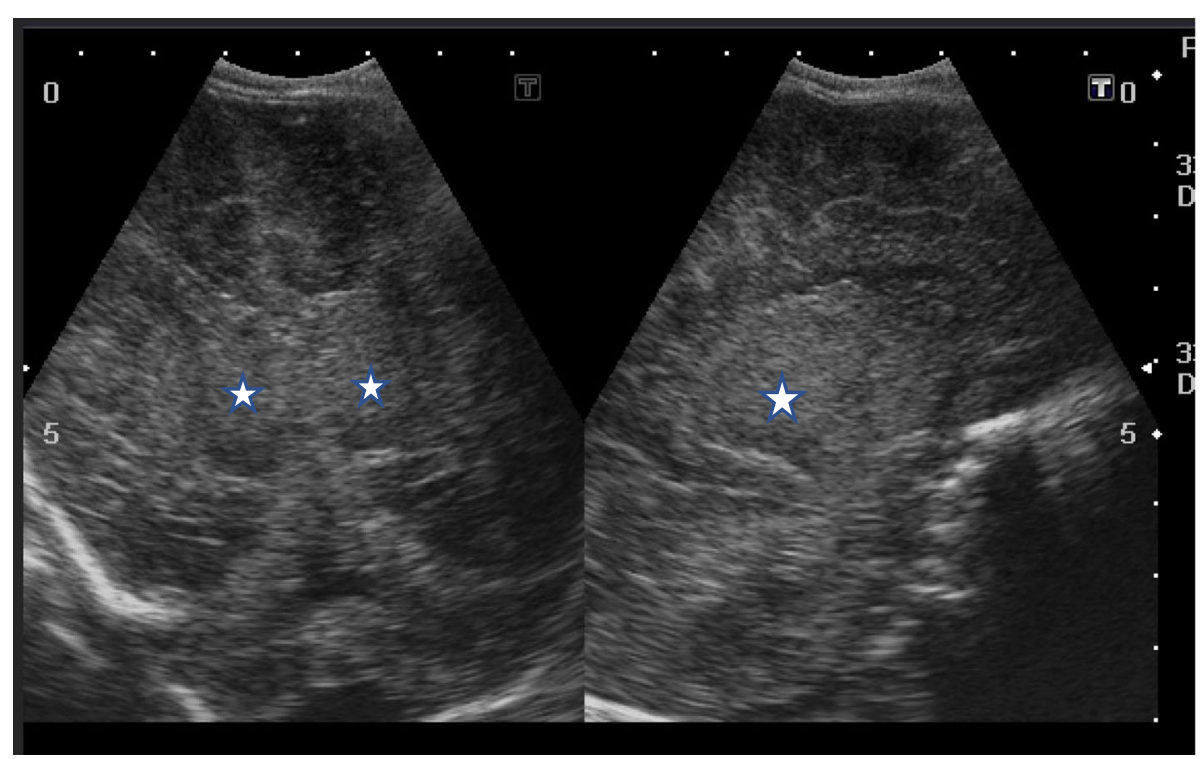

Fig. 7 Coronal and sagittal US image of a neonate with Sarnat stage III showing severe hypoxic ischemic insult in terms of cerebral cortex and bilateral thalamic (white stars) and basal ganglia diffuse increased echogenicity with marked edema and compressed ventricles 


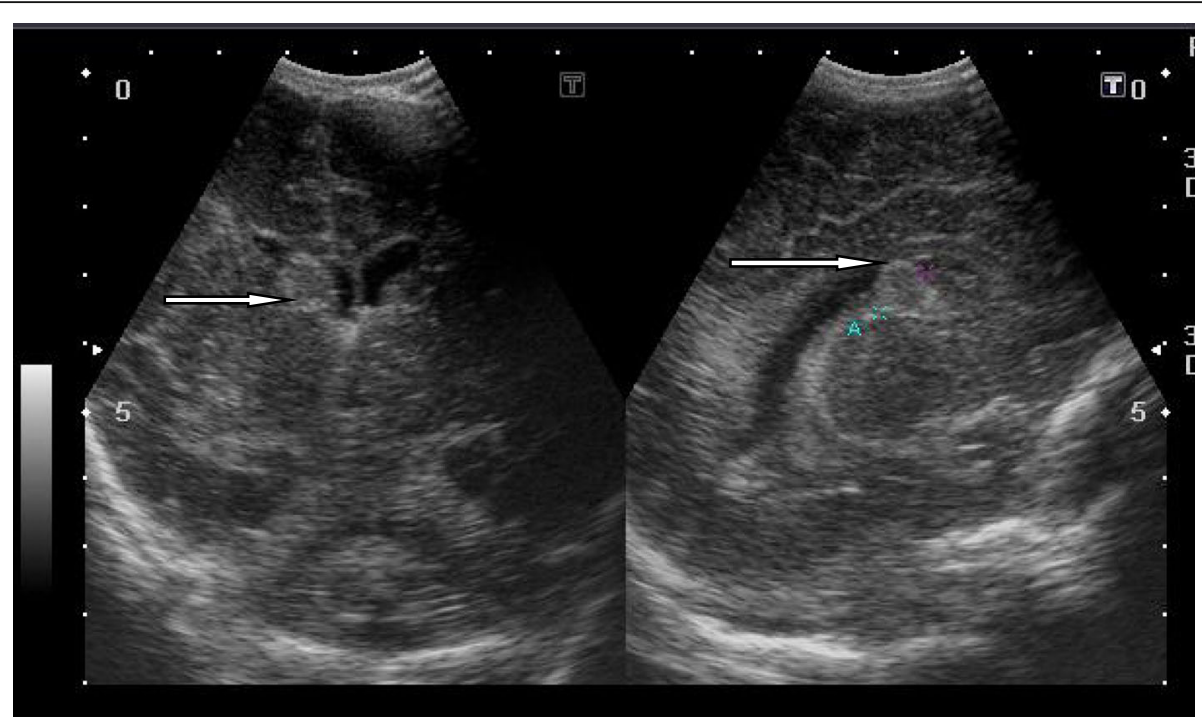

Fig. 8 Coronal and sagittal US of a neonate with Sarnat stage II showing large echogenic subependymal hemorrhage of the right lateral ventricle, extending into the lumen representing grade I periventricular hemorrgae with impending intra ventricular extension

considering the scarcity of the condition, time factor was a restriction.

\section{Conclusion}

Serum MDA sensitivity and specificity was $100 \%$ in hypoxic neonates which makes it a reliable diagnostic marker of oxidative stress in perinatal asphyxia. The significant correlation between Sarnat stages and serum MDA levels also provides us with a prognostic marker for cerebral damage and neurological outcome for neonates with HIE. An important cut-off value for MDA was $>2.88 \mathrm{nmol} / \mathrm{ml}$ which differentiated higher Sarnat stages, earlier onset of seizures, positive cranial ultrasound hypoxic findings, longer hospital stays, and higher chances of mortality.

Even though the sensitivity and specificity of cranial ultrasound for newborns with HIE is weak, it is still considered a noninvasive, relatively low-cost screening tool of the hemodynamically unstable neonate. Ultrasound was found to have low sensitivity in the diagnosis of HIE especially in milder grades. However, combination of ultrasound with measuring MDH concentration could act as diagnostic marker and a predictor of disease severity"
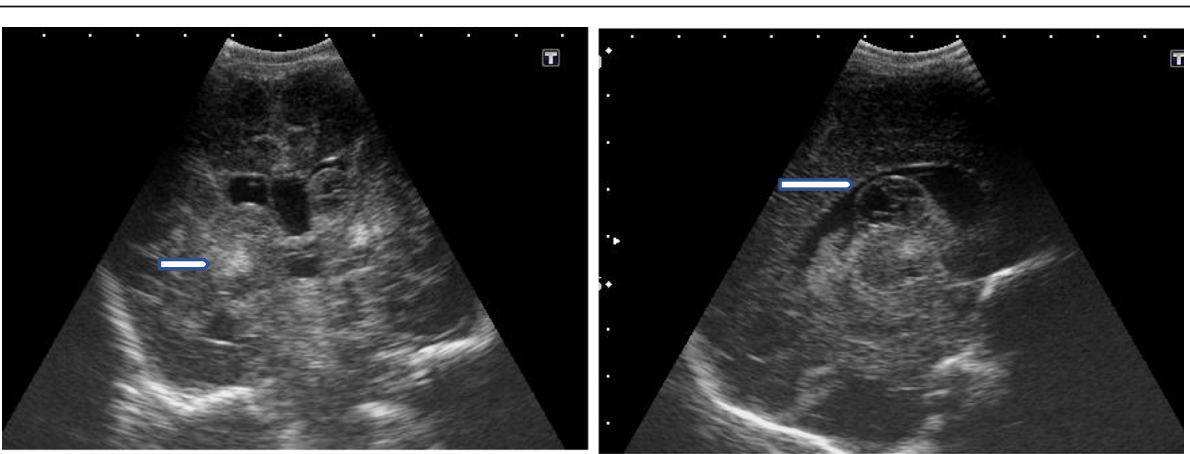

Fig. 9 Coronal and sagittal US of a neonate full term. CS, cyanoses, Apgar scoring 2 and 4 at 1 and 5 min, anteparum hge, mechanical ventilator, and meconium aspiration syndrome. Sarnat stage III. Cranial US revealed aging subependymal hge (arrow), bilateral basal ganglia focal ecchognic areas (arrow head), and severe ischemia 
Table 4 Relation between US evidence of HIE and Sarnat stages

\begin{tabular}{|c|c|c|c|c|c|c|c|}
\hline \multirow[b]{2}{*}{ Sarnat stage } & \multicolumn{2}{|c|}{ HIE by US $(n=11)$} & \multicolumn{2}{|c|}{ No HIE by US $(n=21)$} & \multirow[b]{2}{*}{$x^{2}$} & \multirow[b]{2}{*}{ df } & \multirow[b]{2}{*}{$P$ valuef } \\
\hline & $\mathrm{N}$ & $\%$ & $\mathrm{~N}$ & $\%$ & & & \\
\hline Stage I & 0 & $.0 \%$ & 10 & $47.6 \%$ & 5.782 & 1 & 0.016 \\
\hline Stage ॥ & 4 & $36.4 \%$ & 4 & $19.0 \%$ & & & \\
\hline Stage III & 7 & $63.6 \%$ & 7 & $33.3 \%$ & & & \\
\hline
\end{tabular}

$x^{2}$ chi-squared statistic, $d f$ Degree of freedom

Chi-squared test for trend

Table 5 Relation between MDA and presence of cranial ultrasound evidence of HIE

\begin{tabular}{llllll}
\hline Variable & +ve findings by US $(\boldsymbol{n}=\mathbf{1 1})$ & -ve findings by US $(\boldsymbol{n}=\mathbf{2 1})$ & $\mathbf{T}$ & df & $\boldsymbol{P}$ valueी \\
\hline Serum MDA $(\mathrm{nmol} / \mathrm{ml})$ & $3.64 \pm 0.83$ & $2.84 \pm 0.86$ & 2.511 & 30 & $\mathbf{0 . 0 1 8}$ \\
\hline
\end{tabular}

Data are mean \pm SD

$t \mathrm{~T}$ statistic, $d f$ Degree of freedom

१Unpaired $t$ Test

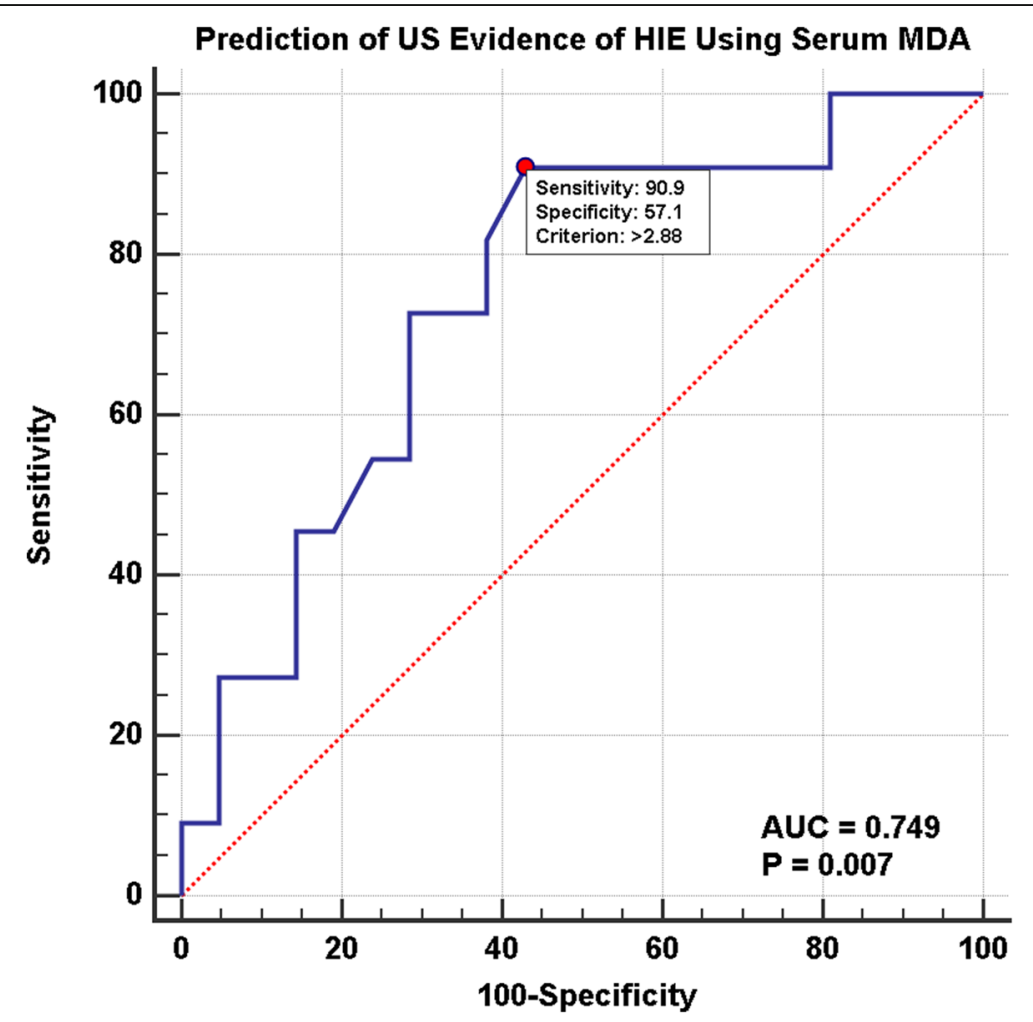

Fig. 10 ROC curve for prediction of cranial US evidence of HIE using serum MDA. There is a highly significant difference between positive and negative US findings using MDA levels $(P=0.007)$. Area under curve was 0.749 . Cut-off MDA in hypoxic neonates with positive US findings was > $2.88 \mathrm{nmol} / \mathrm{ml}$. Sensitivity of MDA in positive US findings was $90.9 \%$ and specificity was $57.14 \%$ 


\section{Abbreviations}

HIE: Hypoxic ischemic encephalopathy; MDH: Malondialdehyde; NICU: Neonatal intensive care unit; US: Ultrasound; PVL: Periventricular leukomalacia; GA: Gestational age

\section{Acknowledgements \\ Not applicable}

\section{Authors' contributions}

Corresponding author: Prof. HM.S.: examining the patients, revising the results, and editing the manuscript. Dr. MM: revising the samples. Prof. ZE: designing the study and revising the manuscript. Prof. N. F: designing the study and revising the manuscript. Dr. MA R: selecting the patients and collecting the data. Dr. S S E: editing and revising the manuscript. All authors have read and approved the manuscript.

\section{Funding}

No funding was received for completion of this study.

\section{Availability of data and materials}

Not applicable

\section{Ethics approval and consent to participate}

The study was conducted over a 2-year duration in accordance with the Declaration of Helsinki. An informed verbal consent was obtained from patients' caregivers, and the study was approved by the local ethical committee (ethical committee of Cairo University). Reference number is not available.

\section{Consent for publication}

Not applicable

\section{Competing interests}

All authors declare that there are no conflicts of interest.

\section{Author details}

'Department of Radiology, Cairo University, Cairo, Egypt. ²Department of Pediatrics, Cairo University, Cairo, Egypt. ${ }^{3}$ Department of Chemical Pathology, Cairo University, Cairo, Egypt.

\section{Received: 5 May 2020 Accepted: 24 November 2020}

Published online: 05 December 2020

\section{References}

1. Shankaran S (2012) Hypoxic-ischemic encephalopathy and novel strategies for neuroprotection. Clin Perinat 39:919-929

2. Weinberger B, Anwar M, Henien S (2004) Association of lipid peroxidation with antenatal betametazone and oxygen radical disorders in preterm infants. Biol Neonate 85(2):121-127

3. Fulia F, Gitto E, Cuzzocrea S (2001) Increased levels of malondialdehyde and nitrite/nitrate in the blood of asphyxiated newborns: reduction by melatonin. J Pineal Res 31:343-349

4. Huang BY, Castillo M (2008) Hypoxic-ischemic brain injury: imaging findings from birth to adulthood. Radiographics 28(2):417-439

5. Volpe JJ (1995) Neurology of the Newborn, 3rd edn. WB Saunders, Philadelphia, pp 21-369

6. Robertson CM, Perlman M (2006) Follow-up of the term infant after hypoxic-ischemic encephalopathy. Paediatr. Child. Health 11:278-282

7. Ballard JL, Khoury JC, Wedig K, Wang L, Eilers-Walsman BL, Lipp R (1991) New ballard score, expanded to include extremely premature infants. J Pediatr 119:417-423

8. Philadelphia. WB Saunders.1995; pp 21 - 369.

9. Epelman M, Daneman A, Kellenberger CJ, Aziz A, Konen O, Moineddin R, White, H.\& Blaser, S. (2010) Neonatal encephalopathy: a prospective comparison of head US and MRI. Pediatr Radiol 40:1640-1650

10. North K, Lowe L (2009) Modern head ultrasound: normal anatomy, variants, and pitfalls that may simulate disease. Ultrasound Clin 4:497-512

11. Qureshi AM, Rehman A, Siddiqi TS (2010) Hypoxic ischemic encephalopathy in neonates. J Ayub Med Coll Abbottabad 22(4):190-3
12. MILSOM I, LADFORS, THIRINGER K. (2002) Influence of maternal, obstetric and fetal risk factors on the prevalence of birth asphyxia at term in a Swedish urban population. Acta Obstet Gynecol Scand 81:909-917

13. Simiyu N, Mchaile N, Katsongeri K, Philemon RN, Msuya E (2017) Prevalence, severity and early outcomes of hypoxic ischemic encephalopathy among newborns at a tertiary hospital, in northern Tanzania. BMC Pediatrics 17:131

14. Alireza S, Shirin M (2019) The Investigation of Rate of Birth Asphyxia and its Relationship with Delivery Mode at Shahid Beheshti Hospital of Isfahan during 2013, 2014, and 2015. Int J Prev Med 10:23

15. Nicole N, Komen W, Ko KH, Mullar C, Obladen M (2001) Early biochemical indicators of hypoxic ischemic encephalopathy after birth asphyxia. Pediatr Res 49(4):502-506

16. Florio P, Luisi S, Moataza B, Torricelli M, Iman I, Hala M (2007) High urinary concentrations of activin $A$ in asphyxiated full-term newborns with moderate or severe hypoxic ischemic encephalopathy. Clin Chem 53(3): 520-522

17. Dede FS, Guney Y, Dede H, Koca C, Dilbaz B, Bilgihan A (2006) Lipid peroxidation and antioxidant activity in patients in labor with nonreassuring fetal status. Eur j obstet Gynecolo Reprod Biol. 124(1):27-31

18. Aliyu I, Lawal TO, Onankpa B (2018) Hypoxic-ischemic encephalopathy and the Apgar scoring system: The experience in a resource-limited setting. J Clin Sci 15(1):18-21

19. Chafer-Pericas C, Cernada M, Rahkonen L, Stefanovic V, Andersson S, Vento M (2016) Preliminary case control study to establish the correlation between novel peroxidation biomarkers in cord serum and the severity of hypoxic ischemic encephalopathy. Free Radic Biol Med 97:244-249

20. Shah S, Kumari P, Goel AK (2014) Clinico biochemical profile of birth asphyxia in neonates of western Odisha. Indian J Child Health 1(3):114-118

21. GUPTA BD, SHARMA P, BALGA J, PARAKH M, SONI JP (2005) Renal failure in asphyxiated neonates. Indian Pediatrics 42:928-934

22. Mohammed LH, Khairy MA, EL-Hussieny NA, Mohammed H Zaazou and Aly RM. Multi-organ dysfunction in neonates with hypoxic-ischemic encephalopathy. Med J Cairo Univ 2010; Vol. 78, No. 1, December 461-467.

23. Kumar A, Ramakrishna SV, Basu S (2008) Oxidative stress in perinatal asphyxia. Pediatr Neurol 38:181-185

24. El Bana SM, Maher SE, Gaber AF, Aly SS (2016) Serum and urinary malondialdehyde (MDA), uric acid, and protein as markers of perinatal asphyxia. Electron Phys 8(7):2614-2619

25. Kirimi E, Peker E, Tuncer O, Hapicioclu H, Narli N, Satar M (2010) Increased serum malondialdehyde level in neonates with HIE: Prediction of disease severity. J Int Med Res 38:220-226

26. Banupriya C (2008) Ratnakar, Doureradjou P, Mondal N, Vishnu B, Koner BC. Can urinary excretion rate of malondialdehyde, uric acid and protein predict the severity and impending death in perinatal asphyxia. Clin Biochem 41(12):968-973

27. Nivedita M, Vishnu Bhat B, C. (2010) Banupriya1 and Bidhan C. Koner. Oxidative stress in perinatal asphyxia in relation to outcome. J Pediatr 77(5): 515-517

28. BlankenbergFG LNN, Bracci P (2000) Sonography CT, and MR imaging: a prospective comparison of neonates with suspected intracranial ischemia and hemorrhage. AJNR Am J Neuroradiol 21:213-218

29. Franco A, Lewis KN (2013) Neonatal cranial ultrasound: current perspectives. Rep Med Imaging 6(1):93

30. Chao CP, Zaleski CG, Patton AC (2006) Neonatal hypoxicischemic encephalopathy: multimodality imaging findings. Radiographics 26:S159_ $\mathrm{S} 172$

31. Van Wezel-Meijler G (2012) Neonatal cranial ultraso $\neg$ nography. Springer 43(1):17

\section{Publisher's Note}

Springer Nature remains neutral with regard to jurisdictional claims in published maps and institutional affiliations. 